
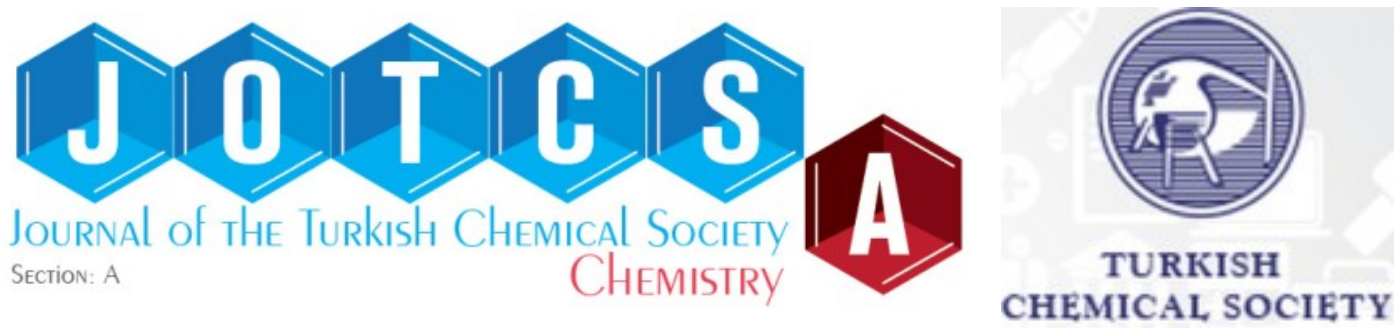

\title{
Effect of Nitrogen Precursor on Optical Properties of Hexagonal Boron Nitride Quantum Dots
}

\author{
Esranur Budak ${ }^{1}$ (D) and Caner Ünlü1,2,3* \\ ${ }^{1}$ Istanbul Technical University, Department of Nanoscience and Nanoengineering, Maslak, 34469 Istanbul, \\ Turkey \\ ${ }^{2}$ Istanbul Technical University, Faculty of Science and Letters, Department of Chemistry, 34469, Maslak, \\ Istanbul, Turkey \\ ${ }^{3}$ Istanbul Technical University Nanotechnology Research and Application Center (ITUNano), 34469, Maslak, \\ Istanbul, Turkey
}

\begin{abstract}
Boron nitride quantum dots (BNQDs) are of high interest with their excellent photophysical and structural characteristics. BNQDs can be synthesized through hydrothermal synthesis methods with different nitrogen precursors; however, until now, the optical properties of BNQDS synthesized with different nitrogen precursors have not been compared in details yet. In this study, BNQDS were synthesized through hydrothermal synthesis methods by using urea, melamine, and thiourea as nitrogen precursors and optical properties of BNQDS were compared by comparing emission and excitation characteristics of each BNQD synthesized by different nitrogen precursor. Structural properties of BNQDs were compared through infrared spectrum of each BNQDs. Our results revealed that the change in nitrogen precursor causes significant differences in photophysical and structural properties of BNQDs.
\end{abstract}

Keywords: Quantum dots, boron nitride, fluorescence.

Submitted: June 29, 2021. Accepted: August 17, 2021.

Cite this: Budak E, Ünlü C. Effect of Nitrogen Precursor on Optical Properties of Hexagonal Boron Nitride Quantum Dots. JOTCSA. 2021;8(3):969-76.

DOI: https://doi.org/10.18596/jotcsa.959577.

*Corresponding author. E-mail: canerunlu@itu.edu.tr.

\section{INTRODUCTION}

Quantum dots made of 2-dimensional materials, in other words graphene like materials, are called 2D quantum dots and have become the center of attention with their excellent photophysical and structural properties (1-4). 2D quantum dots have a lateral size in the range of $2-6 \mathrm{~nm}$ with thickness less than $1 \mathrm{~nm}$ and display graphene-like geometry (1-4). These nanomaterials are simply nanometersized blocks of well-known 2D materials such as graphene, graphitic carbon nitride, hexagonal boron nitride, molybdenum sulfide, molybdenum selenide, tungsten sulfide, etc (1-4). 2D quantum dots have been considered as versatile materials since their discovery and have been used in many different application and research areas such as optoelectronics, supercapacitors, batteries, cell imaging, and sensors (1-7).
Amongst all, BNQDs have been studied widely with their unique features such as low toxicity and high biocompatibility (1-4). BNQDs were discovered in 2014 by Lin et al. and firstly synthesized through reducing the size of bulk hexagonal boron nitride (top-down approach) (8). After their discovery, BNQDs have been synthesized through top-down synthesis methods (8-16) and bottom-up synthesis methods $(15,17-19)$ in many different studies. In most of studies in which bottom-up synthesis methods were used, boric acid was used as the boron precursor; however, the nitrogen precursor was different (15,17-19). The most common used nitrogen precursors were ammonia, urea, and melamine (15,17-19). Each study claimed the final product that they obtained were BNQDs, and also the spectral outcomes were pointing out the quantum dots were indeed similar in structure, however, no detailed comparison on optical 
properties of BNQDs synthesized with different nitrogen precursor was conducted until now.

In this study, we synthesized BNQDs through hydrothermal bottom-up synthetic methods by using boric acid as the boron precursor and 3 different nitrogen precursors; urea, melamine, and thiourea. The photophysical properties of BNQDs were analyzed in details by collecting emission and excitation spectrum of each quantum dot type. Detailed structural analysis was conducted by using Fourier transform infrared spectroscopy. Our results showed that each BNQD type had its unique optical and structural properties and therefore they should be considered as different quantum dot type.

\section{MATERIALS AND METHODS}

All chemicals that were used in this work were of the highest purity and were purchased from SigmaAldrich Co. They were used without further purification.

\section{Synthesis of Boron Nitride Quantum Dots}

Boron nitride (BN) quantum dots were synthesized through a modified hydrothermal synthesis method (17). To synthesize BN quantum dots, boric acid was used as a boron precursor and three different nitrogen precursors (urea, thiourea, and melamine) were selected to accompany boric acid. Firstly, 300 $\mathrm{mg}$ of boric acid and $100 \mathrm{mg}$ of nitrogen precursor were completely dissolved in $5 \mathrm{~mL}$ of distilled water. Then, the clear solution was transferred into a Teflon-lined stainless steel reaction chamber, sealed, and placed in a furnace oven (brand: Isolab). The reaction chamber was heated until 200 ${ }^{\circ} \mathrm{C}$ and kept at that temperature for 24 hours. After 24 hours, the reaction chamber was taken out and cooled down to room temperature. A colorless clear solution was obtained as the crude product, which was purified by filtering with 0.22 micrometer syringe and centrifuging at $14,000 \mathrm{rpm}$ for 30 minutes. The purified solution was kept at $4{ }^{\circ} \mathrm{C}$ for further use.

\section{Optical and Structural Characterization of C-BN and BCN QDs}

Optical properties of BN QDs were characterized by Scinco Neosys-2000 single - beam ultraviolet-visible (UV-Vis) spectrophotometer and Varian Cary Eclipse fluorescence spectrofluorimeter. Each sample was dissolved in $10 \mathrm{~mL}$ of ultrapure water after purification and diluted until optical density was under 0.1 in order to prevent errors resulting from self-absorption, then the absorption spectrum of each sample was collected by a UV-Vis spectrophotometer. The further optical properties were determined through aqueous solutions of quantum dots. Emission spectrum of each sample was collected by using different excitation wavelengths $\left(\lambda_{\text {exc }}\right)$ in the range of $250-400 \mathrm{~nm}$. The emission color of each sample was observed under 366 nm UV-light. Photoluminescent excitation (PLE) spectrum of each sample was collected at 440 $\mathrm{nm}$. Quantum yields of the BN QDs were measured by using coumarin 102 in ethanol as a standard (the reference quantum yield was 0.93$)(20,21)$.

Structural analysis of BN quantum dots was performed through Fourier transform infrared spectroscopy (FTIR) and Transmission electron microscopy (TEM). Bonding characteristics of BN quantum dots were determined by attenuated total reflectance FTIR spectroscopy. FTIR spectrum of each quantum dot was carried out in the range of $3750-800 \mathrm{~cm}^{-1}$ by using a Perkin Elmer Spectrum One ATR-FTIR spectrometer.

The shape and size of quantum dots were determined by using TEM. TEM measurements were conducted with JEOL JEM 1220 at an acceleration voltage of $100 \mathrm{kV}$.

\section{RESULTS AND DISCUSSION}

\section{Synthesis of BN quantum dots}

Bottom-up synthesis of BN QDs are generally carried out by using various boron and nitrogen precursors $(15,17-19)$. In most of the studies, even though the precursors for boron and nitrogen differ, the synthesis conditions are similar to synthesize BN quantum dots (15,17-19). In this study, boric acid was chosen to be the sole boron precursor (Figure 1). As nitrogen precursor, melamine, urea, or thiourea were used with boric acid separately to synthesize BN QDs in order to check the effect of nitrogen precursor on structural and optical properties of BN QDs. The duration for synthesis and synthesis temperature were chosen to be 24 hours and $200{ }^{\circ} \mathrm{C}$, respectively, as these conditions were frequently used to synthesize BN QDs (Figure 1) $(15,17-19)$.

As the molecular structure of nitrogen precursors were compared, it was seen that in fact all these precursors can also act as precursor of additional elements, such as sulfur, oxygen, and carbon (Figure 2). Urea and melamine are frequently used in synthesis of BN QDs, however, thiourea has not been preferred widely (15,17-19). In the literature, it was suggested that BN QDs can be synthesized through using either melamine or urea, however, it should be considered that different molecular structures of urea and melamine most likely cause a distinct difference in the structure of QDs, therefore in the optical properties of QDs. 


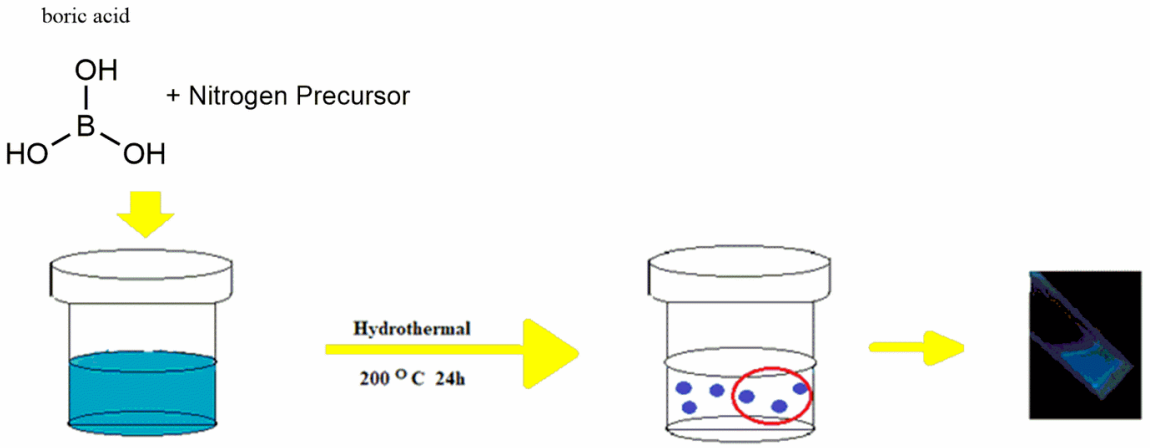

Figure 1. Schematic representation of synthesis of Boron Nitride quantum dots.<smiles>NC(N)=O</smiles>

urea<smiles>NC(N)=S</smiles>

thiourea<smiles>Nc1nc(N)nc(N)n1</smiles>

melamine

Figure 2. Structures of nitrogen precursors.

\section{Structural characterization of BN QDs}

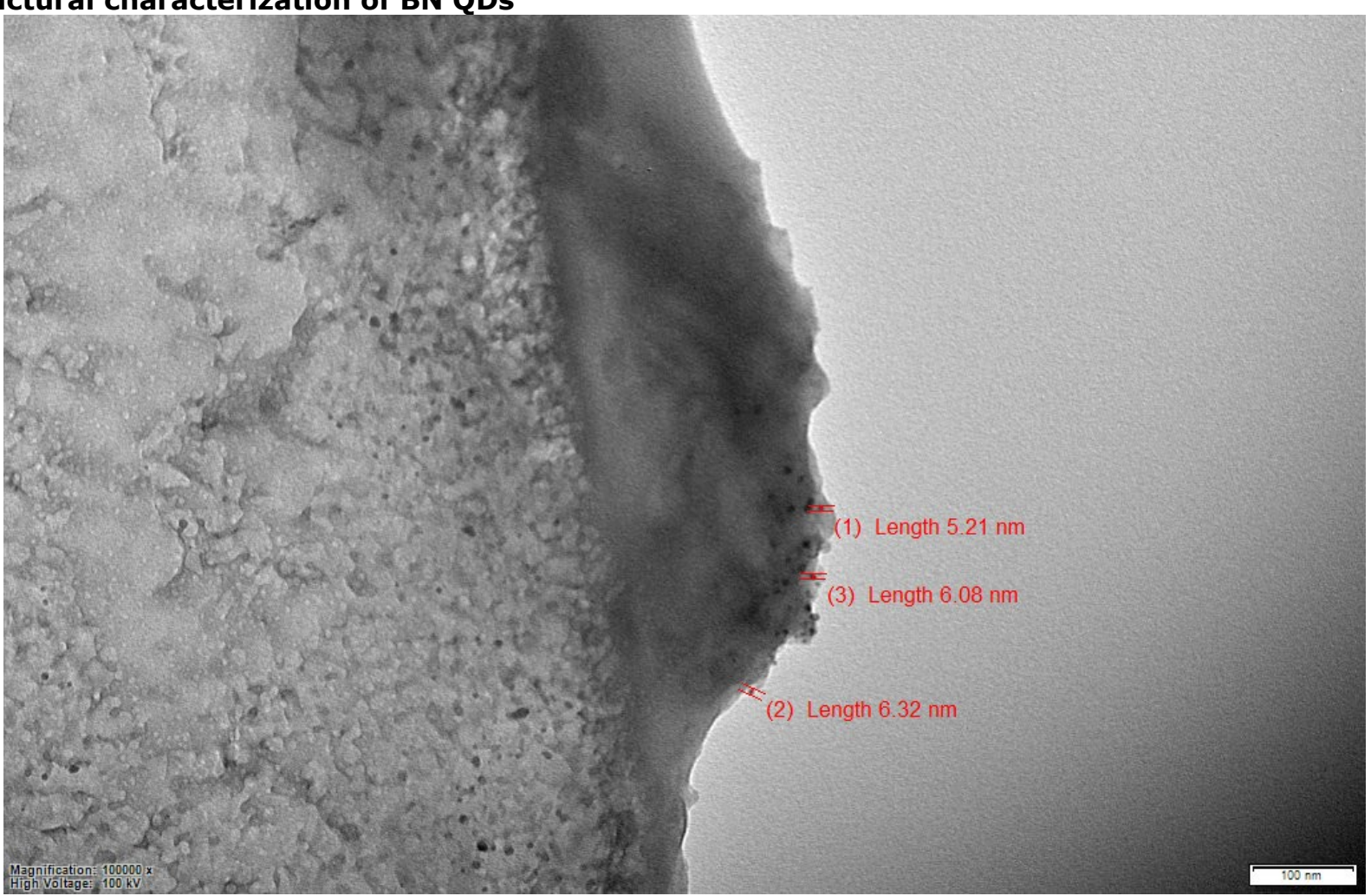

Figure 3. TEM image of BNQDs.

The size characterization was performed on $U$ BNQDs by TEM and it was found out that BN QDs had a size around $2-5 \mathrm{~nm}$, which is the typical size range for BNQDs (Figure 3). The structural differences in BN QDs synthesized by different nitrogen precursors displayed themselves in FTIR spectrum of each QD (Figure 4). The most observable difference was explained as follows; BN QDs derived from thiourea (T-BNQDs) had a very sharp and intense peak at $2550 \mathrm{~cm}^{-1}$ which corresponded to S-H bonding where neither BN QDs derived from urea (U-BNQDs) nor BN QDs derived from melamine (M-BNQDs) had (Figure 4) (15,1719). This peak showed that $S=C$ double bond in 
thiourea was broken during synthesis of BN QDs and thiol groups were formed in the structure of $T$ BNQDs. FTIR spectrum of U-BNQDs and M-BNQDs had typical $-\mathrm{BOH}$ bonding peaks around $1180 \mathrm{~cm}^{-1}$ however, this peak shifted to $1210 \mathrm{~cm}^{-1}$ in FTIR spectrum of T-BNQDs due to existence of $-\mathrm{SH}$ groups on the surface of T-BNQDs (Figure 4) (15,17-19). Each BN QD had typical B-N bonding peak around $780-800 \mathrm{~cm}^{-1}$ and $1380-1420 \mathrm{~cm}^{-1}$ (Figure 4). As the structures of U-BNQDs and $\mathrm{M}$ BNQDs were compared, the main difference between the FTIR spectra of U-BNQDs and $M$ BNQDs was the peak around $920 \mathrm{~cm}^{-1}$, the $-\mathrm{N}-\mathrm{B}-\mathrm{O}$ edge peak, which was the clear evidence of structural difference between U-BNQDs and $M$ BNQDs (Figure 4). Adjacent to the peak at $880 \mathrm{~cm}^{-1}$, attributed to stretching vibration bands of $\mathrm{C}-\mathrm{O}$, the U-BNQDs possessed a peak at $920 \mathrm{~cm}^{-1}$ which showed that U-BNQDs had N-B-O groups on the surface (Figure 4). These clear differences showed that depending on nature of nitrogen precursor, BN QDs had different structural characteristics.

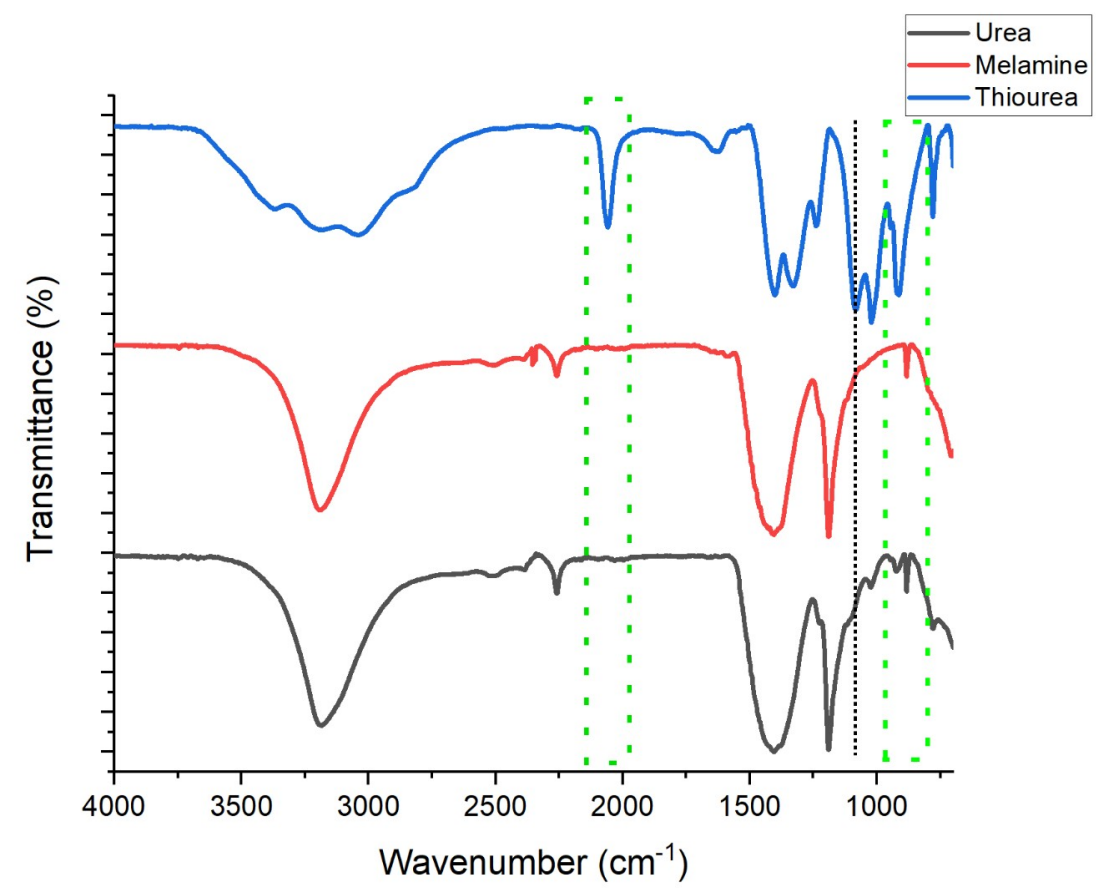

Figure 4. FTIR spectra of BNQDS synthesized with urea (black), melamine (red) and thiourea (blue).

\section{Optical characterization of BN QDs}
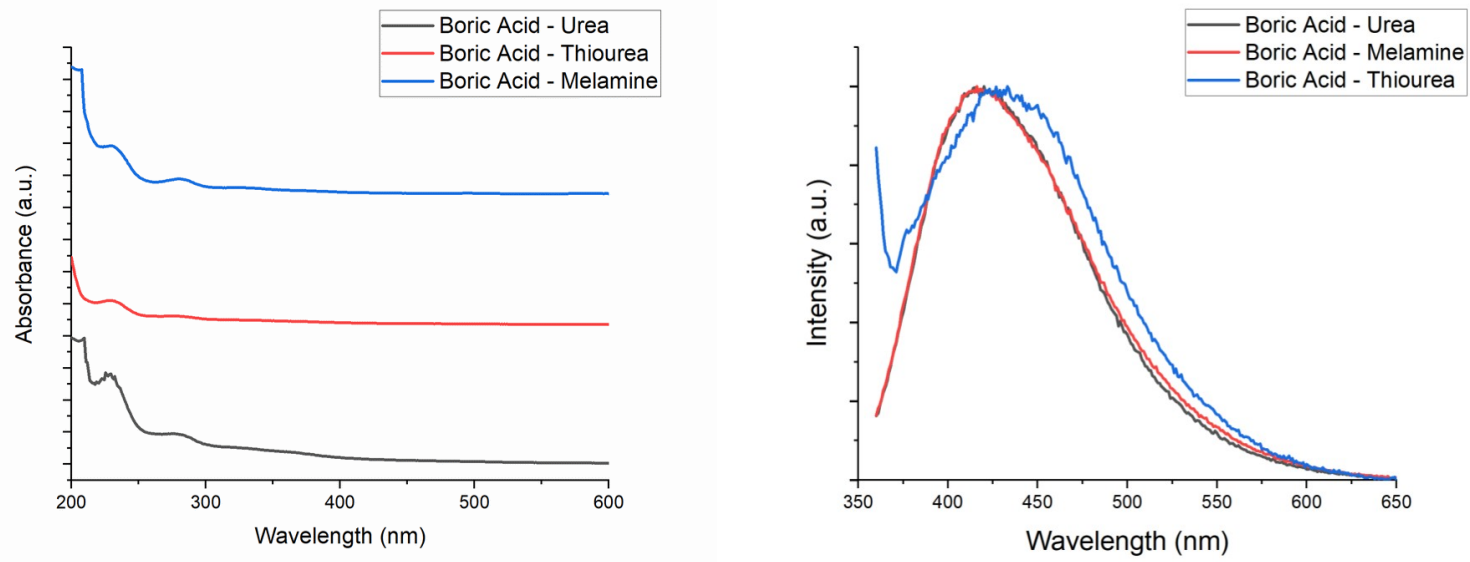

Figure 5. Absorption (left) and Fluorescent spectrum (right) of BNQDS synthesized with urea (black), melamine (blue), and thiourea (red). For fluorescent spectrum, each BNQDs were excited by $350 \mathrm{~nm}$ light.

Absorption spectrum and emission (PL) spectrum of a quantum dot are the two main indicators that let us characterize optical properties of all types of quantum dots $(15,17-19)$. The comparison of absorbance spectrum of each BN QDs revealed that the absorption characteristics of M-BNQDs and $U$ BNQDs were similar, where absorbance spectrum of T-BNQDs was different from those of U-BNQDs and 
M-BNQDs (Figure 5). Absorbance spectra of $U$ BNQDs and M-BNQDs possessed an intense peak around $230 \mathrm{~nm}$, due to $\mathrm{C}=\mathrm{C}$ peak in the structure of BN QDs and a relatively mild peak around $280 \mathrm{~nm}$, due to band-edge transition in BN QDs (Figure 5) (15,17-19). Both of these peaks also appeared in TBNQDs, but with a dramatically low intensity, which showed that the structure of T-BNQDs was considerably different from structure of M-BNQDs and U-BNQDs, as was also indicated through FTIR spectrum of each BN QDs. The emission spectrum of each BN QDs revealed the same story; emission spectrum of M-BNQDs upon $\lambda_{\text {exc }}=350 \mathrm{~nm}$ and emission spectrum of U-BNQDs upon $\lambda_{\text {exc }}=350 \mathrm{~nm}$ were almost identical, a single peak around $416 \mathrm{~nm}$, where the emission peak of T-BNQDs red-shifted almost $15 \mathrm{~nm}$, a single peak around $432 \mathrm{~nm}$ (Figure $5)$. The incorporation of $S$ into $B N$ QD structure caused a considerable red-shift in emission peak of BN QDs, and also caused a significant decrease in quantum yield of $B N$ QDs; the U-BNQDs and $M-$ BNQDs had quantum yield of $24 \%$ and $21 \%$, respectively, where T-BNQDs had quantum yield of $6 \%$. Although the FTIR spectrum of M-BNQDs and U-BNQDs were different, the absorption and emission spectrum were quite similar. Yet, the further analysis on optical properties of BN QDs showed that M-BNQDs and U-BNQDs had different excitation properties (Figures 6, 7, and 8).

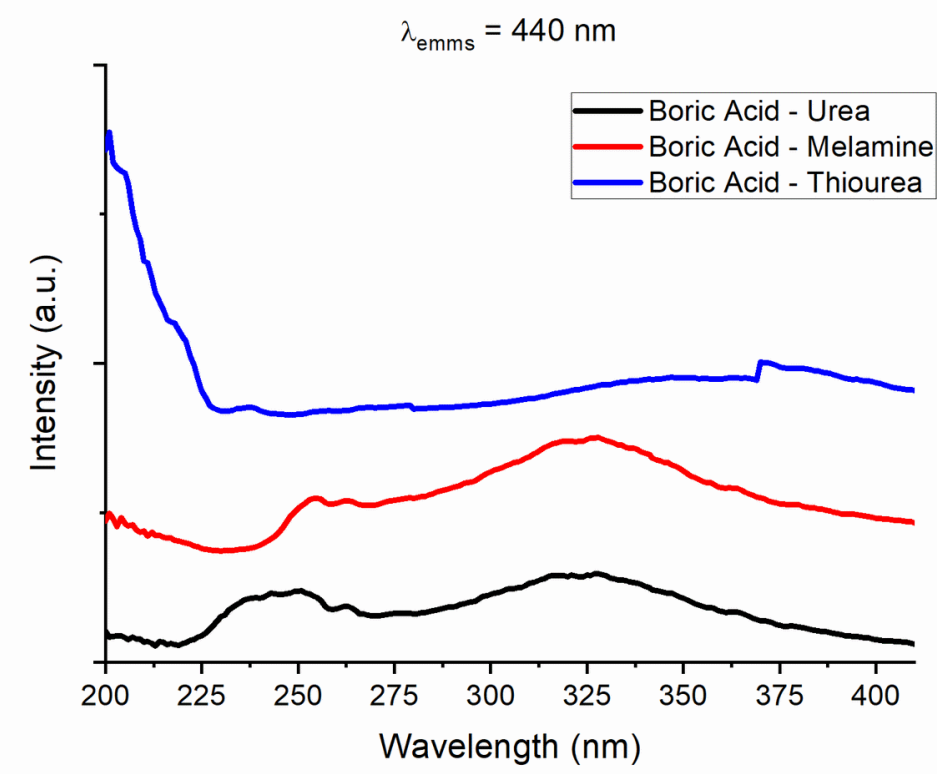

Figure 6. PLE spectrum of BNQDS synthesized with urea (black), melamine (red), and thiourea (blue).

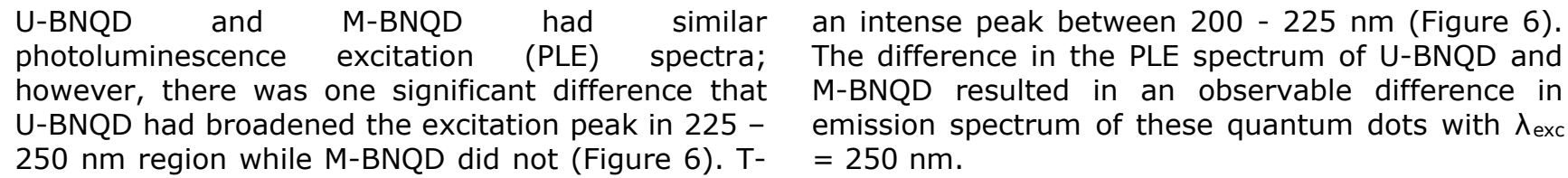




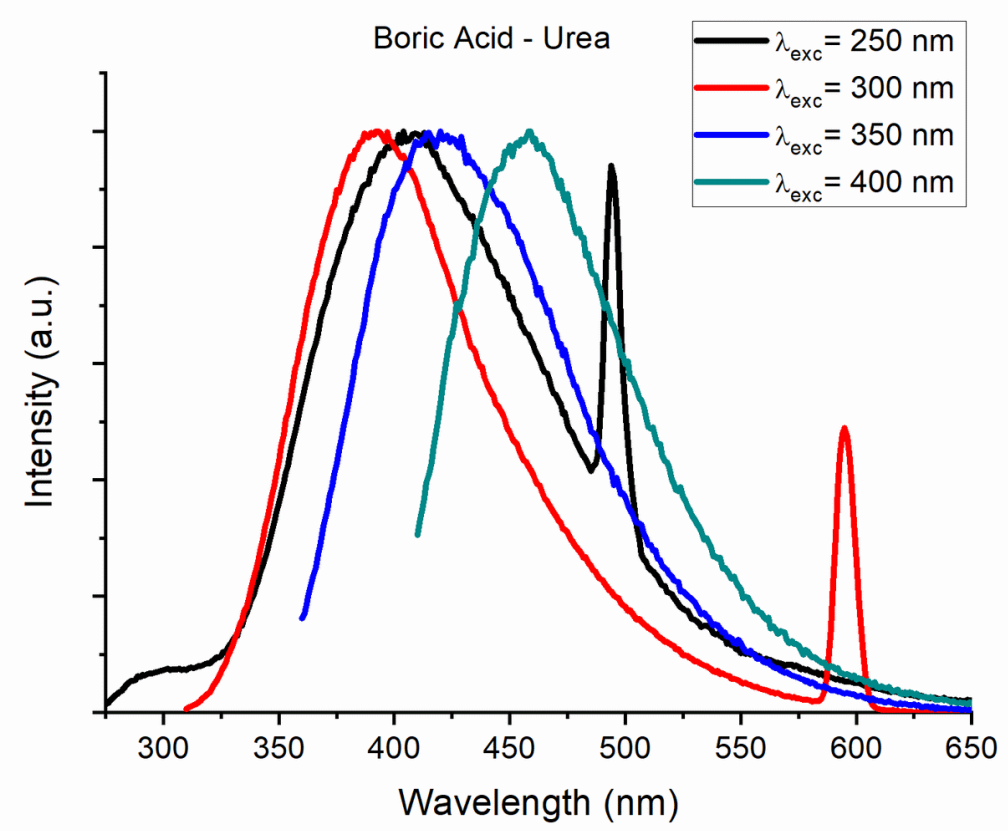

Figure 7. Fluorescent spectra of BNQDS synthesized with melamine with different excitation wavelengths.

The emission spectrum of M-BNQD had a single peak at $415 \mathrm{~nm}$ with $\lambda_{\text {exc }}=250 \mathrm{~nm}$ (Figure 7) where the emission spectrum of U-BNQD had a single peak at $395 \mathrm{~nm}$ with $\lambda_{\text {exc }}=250 \mathrm{~nm}$ (Figure 8 ). This difference is most likely due to existence of different groups on the surface of M-BNQD and $U$ -
BNQD, the existence N-B-O groups on the surface of $U-B N Q D s$. It should be noted that U-BNQD and $M-$ BNQD had very similar emissive properties with $\lambda_{\text {exc }}$ $=300 \mathrm{~nm}, \lambda_{\text {exc }}=350 \mathrm{~nm}$ and $\lambda_{\text {exc }}=400 \mathrm{~nm}$ (Figures 7 and 8).

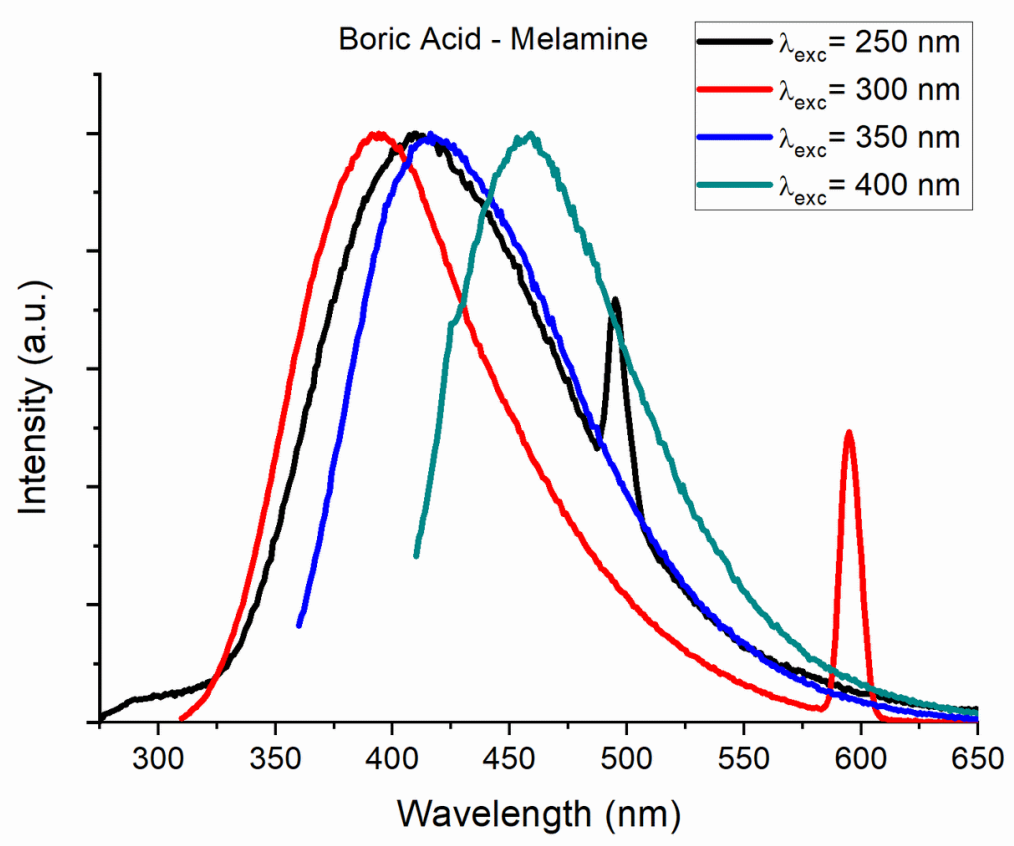

Figure 8. Fluorescent spectrum of BNQDS synthesized with urea with different excitation wavelengths.

These observations showed that, despite the differences on the surface of U-BNQD and M-BNQD, these quantum dots had similar inner crystal structure and the crystal structure can be excited through lower energy possessing light (300 - 400 $\mathrm{nm})$, where surface of BN QDs could be excited through higher energy possessing light (250 nm). 


\section{CONCLUSION}

Boron nitride quantum dots are generally synthesized through hydrothermal methods by using boric acid as a boron precursor and various chemicals such as urea, melamine, and thiourea as nitrogen precursors. The BN quantum dots synthesized with different nitrogen groups had significant optical and structural differences. When thiourea was used as nitrogen precursor, it was observed that sulfur was incorporated with crystal structure and incorporation of sulfur to nanocrystal structure caused significant changes in optical properties of quantum dots, such as decrease in quantum yield and shift in emission peak wavelength. The BN quantum dots synthesized by using urea and melamine as nitrogen precursors had similar emission properties upon excitation through 300, 350, and $400 \mathrm{~nm}$ light, however, the U-BNQD had a broadened PLE spectrum compared to M-BNQD and had different emission properties upon excitation through $250 \mathrm{~nm}$, which was a consequence of existence of $\mathrm{N}-\mathrm{B}-\mathrm{O}$ bonding on the surface of U-BNQD that was observed in FTIR spectrum of U-BNQD.

\section{ACKNOWLEDGEMENT}

We would like to thank Dr. Bünyamin Karagöz for letting use of fluorescent spectrophotometer. Also, we thank Dr. Ahmet Gül for helpful discussion, support, and letting use of UV-Vis spectrophotometer. This work was supported by TUBITAK (The Scientific and Technological Research Council of Turkey) under Career Development Programme for Young Researchers (Career Programme) (Programme No: 3501) [Grant number: 118Z259].

\section{REFERENCES}

1. Wang X, Sun G, Li N, Chen P. Quantum dots derived from two-dimensional materials and their applications for catalysis and energy. Chem Soc Rev. 2016;45(8):2239-62. <DOI .

2. Xu Y, Wang X, Zhang WL, Lv F, Guo S. Recent progress in two-dimensional inorganic quantum dots. Chem Soc Rev. 2018;47(2):586-625. <DOI>.

3. Xu Q, Cai W, Li W, Sreeprasad TS, He Z, Ong WJ, et al. Two-dimensional quantum dots:

Fundamentals, photoluminescence mechanism and their energy and environmental applications. Materials Today Energy. 2018 Dec;10:222-40. $<$ DOI $>$.

4. Manikandan A, Chen $Y-Z$, Shen C-C, Sher C-W, Kuo $\mathrm{H}-\mathrm{C}$, Chueh $\mathrm{Y}-\mathrm{L}$. A critical review on twodimensional quantum dots (2D QDs): From synthesis toward applications in energy and optoelectronics. Progress in Quantum Electronics. 2019 Nov;68:100226. <DOI>.

5. Budak $E$, Ünlü $C$. Boron regulated dual emission in $\mathrm{B}, \mathrm{N}$ doped graphene quantum dots. Optical Materials. 2021 Jan;111:110577. <DOI>.

6. Budak E, Aykut S, Paşaoğlu ME, Ünlü C. Microwave assisted synthesis of boron and nitrogen rich graphitic quantum dots to enhance fluorescence of photosynthetic pigments. Materials Today Communications. 2020 Sep;24:100975. <DOI>.

7. Budak E, Erdoğan D, Ünlü C. Enhanced fluorescence of photosynthetic pigments through conjugation with carbon quantum dots. Photosynth Res. 2021 Jan;147(1):1-10. <DOI>.

8. Lin $L, X u Y$, Zhang $S$, Ross IM, Ong ACM, Allwood DA. Fabrication and Luminescence of Monolayered Boron Nitride Quantum Dots. Small. 2014 Jan $15 ; 10(1): 60-5$. <DOI .

9. Pan C, Long M, He J. Enhanced thermoelectric properties in boron nitride quantum-dot. Results in Physics. 2017;7:1487-91. <DOI>

10. Fan L, Zhou $Y$, He $M$, Tong $Y$, Zhong $X$, Fang J, et al. Facile microwave approach to controllable boron nitride quantum dots. J Mater Sci. 2017 Dec; 52(23):13522-32. <DOI>.

11. Matsoso BJ, Ranganathan K, Mutuma BK, Lerotholi T, Jones G, Coville NJ. Single-step synthesis of crystalline h-BN quantum- and nanodots embedded in boron carbon nitride films. Nanotechnology. 2017;28(10):105602. <DOI>.

12. Thangasamy $P$, Santhanam $M$, Sathish $M$. Supercritical Fluid Facilitated Disintegration of Hexagonal Boron Nitride Nanosheets to Quantum Dots and Its Application in Cells Imaging. ACS Appl Mater Interfaces. 2016 Jul;8(29):18647-51. $\leq$ DOI $>$.

13. Bandyopadhyay A, Yamijala SSRKC, Pati SK. Tuning the electronic and optical properties of graphene and boron-nitride quantum dots by molecular charge-transfer interactions: a theoretical study. Phys Chem Chem Phys. 2013;15(33):13881. $\leq$ DOI $>$.

14. Li H, Tay RY, Tsang SH, Zhen X, Teo EHT. Controllable Synthesis of Highly Luminescent Boron Nitride Quantum Dots. Small. 2015

Dec;11(48):6491-9. <DOI>.

15. Yang $Y$, Zhang $C$, Huang D, Zeng G, Huang J, Lai $C$, et al. Boron nitride quantum dots decorated ultrathin porous g-C3N4: Intensified exciton dissociation and charge transfer for promoting visible-light-driven molecular oxygen activation. 
Applied Catalysis B: Environmental. 2019

May;245:87-99. <DOI $>$.

16. Stampfl J, Tasch S, Leising G, Scherf U. Quantum efficiencies of electroluminescent poly(para-phenylenes). Synthetic Metals. 1995 Apr;71(1-3):2125-8. <DOI>.

17. Yao Q, Feng Y, Rong M, He S, Chen X. Determination of nickel(II) via quenching of the fluorescence of boron nitride quantum dots. Microchim Acta. 2017 Oct; 184(10):4217-23. $\leq$ DOI $>$.

18. Huo B, Liu B, Chen T, Cui L, Xu G, Liu M, et al. One-Step Synthesis of Fluorescent Boron Nitride Quantum Dots via a Hydrothermal Strategy Using Melamine as Nitrogen Source for the Detection of
Ferric Ions. Langmuir. 2017 Oct 10;33(40):106738. $<$ DOI $>$.

19. Liu $M, X u Y$, Wang $Y$, Chen $X$, Ji X, Niu F, et al. Boron Nitride Quantum Dots with Solvent-Regulated Blue/Green Photoluminescence and

Electrochemiluminescent Behavior for Versatile Applications. Advanced Optical Materials. 2017 Feb;5(3):1600661. <DOI $>$.

20. Liu B, Yan S, Song Z, Liu M, Ji X, Yang W, et al. One-Step Synthesis of Boron Nitride Quantum Dots: Simple Chemistry Meets Delicate Nanotechnology. Chem Eur J. 2016 Dec 23;22(52): 18899-907. $\leq \mathrm{DOI}$.

21. Fletcher AN, Bliss DE. Effects of chemical substituents of bicyclic dyes upon photodegradation parameters. Appl Phys. 1978;16:289. 